\title{
CONGESTION EFFECTS OF AUTONOMOUS TAXI FLEETS
}

\author{
Michal MACIEJEWSKI ${ }^{1^{*}}$, Joschka BISCHOFF${ }^{2}$ \\ ${ }^{1}$ Division of Transport Systems, Poznan University of Technology, Poland \\ ${ }^{2}$ Dept of Land and Sea Transport Systems, Technical University of Berlin, Germany
}

Received 13 June 2016; revised 23 October 2016, 31 January 2017; accepted 6 February 2017; published online 4 September 2017

\begin{abstract}
Fleets of shared Autonomous Vehicles (AVs) could replace private cars by providing a taxi-like service but at a cost similar to driving a private car. On the one hand, large Autonomous Taxi (AT) fleets may result in increased road capacity and lower demand for parking spaces. On the other hand, an increase in vehicle trips is very likely, as travelling becomes more convenient and affordable, and additionally, ATs need to drive unoccupied between requests. This study evaluates the impact of a city-wide introduction of ATs on traffic congestion. The analysis is based on a multi-agent transport simulation (MATSim) of Berlin (Germany) and the neighbouring Brandenburg area. The central focus is on precise simulation of both real-time AT operation and mixed autonomous/conventional vehicle traffic flow. Different ratios of replacing private car trips with AT trips are used to estimate the possible effects at different stages of introducing such services. The obtained results suggest that large fleets operating in cities may have a positive effect on traffic if road capacity increases according to current predictions. ATs will practically eliminate traffic congestion, even in the city centre, despite the increase in traffic volume. However, given no flow capacity improvement, such services cannot be introduced on a large scale, since the induced additional traffic volume will intensify today's congestion.
\end{abstract}

Keywords: autonomous vehicle, autonomous taxi, taxi dispatching, traffic flow, queue model, large-scale simulation, MATSim.

\section{Introduction}

Autonomous Vehicles (AVs) are often considered a solution to the current problems of providing convenient, safe, efficient and still affordable ways of travelling. There is a lot of speculation of how AVs will change people's travel behaviour and what will be the impact on today's transport systems. Although numerous studies have tried to roughly estimate the possible effects of introducing AVs (Wadud et al. 2016; Litman 2015), we are still far from having clear answers. However, it is beyond question that the possibility of relocating empty AVs will ease sharing them among many people. Because sharing private AVs on a small scale, within families or small circles of people, imposes the need of synchronisation among users, only large-scale Autonomous Taxi (AT) services can eliminate such constraints and allow taking full advantage of the AV technology.

AT services are aimed at combining advantages of different modes of transport, such as private car, shared car and taxi. From the traveller's point of view, they can be considered a taxi-like service but at a cost similar to driving a private car, hence the demand for AT trips is expected to be at a level of today's car trips demand. On the fleet level, such services can help us greatly reduce the number of vehicles and parking spaces; nowadays private cars stay idle for almost the whole day. However, it remains unclear what impact AT fleets will have on traffic. Besides induced travel demand caused by higher accessibility and affordability, which is not dealt with in this study, large AT fleets may result in:

- increased road capacity resulting from high share of AVs;

- higher traffic volumes due to unoccupied drives between requests.

This study investigates how an increased capacity and higher volumes, both being direct effects of a citywide introduction of ATs, will impact overall traffic. The analysis is based on a Multi-Agent Transport Simulation (MATSim) of Berlin, the 3.5 million capital city of Germany, and the neighbouring Brandenburg area. The central focus is on precise simulation of both real-time AT operation and mixed autonomous/conventional vehicle traffic flow. We use MATSim (Horni et al. 2016b;

${ }^{\star}$ Corresponding author. E-mail: michal.maciejewski@put.poznan.pl 
Kickhöfer et al. 2016) to simulate a complete workday in Berlin with a high-detail disaggregated demand (individual travellers and their trips) and a large AT fleet being dispatched in real time in response to incoming events (such as request submission or vehicle arrival). The study covers different stages of the AT introduction, ranging between 0 and $100 \%$ of private car trips being converted into AT ones.

The study is built upon previous works on realtime taxi dispatching simulation and the case study of AT services in Berlin, referenced later in the paper. The contribution of this paper is as follows:

- we extended the queue model available in MATSim with AV vehicles, so that mixed traffic, consisting of autonomous and conventional vehicles can be simulated. This allowed us to run simulations for different shares of ATs and different levels of road capacity increase;

- we combined the standard user-optimum relaxation scheme available in MATSim with centralised AT dispatching. By using long-term travel time averages for AT dispatching, we were able to avoid day-to-day oscillations and relatively quickly achieve a dynamic equilibrium;

- we ran large-scale simulations for different shares of AT trips and different road capacity increase levels and carried out a comprehensive comparison that included many different indicators measuring effects on congestion and the AT service itself. Even a moderate improvement of capacity turned out to be positive for both congestion reduction and AT service performance.

\section{State-of-the-art}

Recent research in AV development has been going on in several fields of science. On one hand, there is actual vehicle-based research, focussing on e.g. building $\mathrm{AVs}$ and allowing them to collaborate via car-to-car and car $2 \mathrm{x}$ communication, which plays a key role in improving overall traffic flow using AVs. On the other hand, there is plenty of research regarding the possible implications of AV availability on mobility patterns and habits of people.

In the first category, several studies suggest a significant improvement of traffic flow in many areas: During cruise, gaps between following vehicles may be as low as $0.3-0.5 \mathrm{~s}$ (Wagner 2015). Taking into account a high penetration rate of cooperative and adaptive cruise control mechanism, the efficiency of such systems in high traffic density has also been demonstrated (Van Arem et al. 2006). At intersections, the throughput of vehicles may be doubled using car2x technology. More precisely, a reservation system for intersections may significantly reduce waiting times (Fajardo et al. 2011).

In the second category, due to the easiness of sharing AVs, AT services are most likely to arise. Depending on the region and living circumstances, owning a vehicle may become far less attractive than it is today, or may even be restricted. In the US, operating costs for an AT system may be as low as $0.15 \$$ per mile (Burns, Scarborough 2013). As to the question, how many Conventionally Driven Vehicles (CDVs) could be replaced by one AT, the numbers differ significantly depending on the operating scheme (shared/non-shared rides), the region involved and whether public transport is also replaced. In Lisbon, for instance, a single AT can replace up to ten cars if rides are shared and only six otherwise (Martínez 2015). However, when also public transport is converted into AT services and ride sharing is not available, much larger AT fleets are necessary, and consequently the replacement ratios are 1:4 for Lisbon (Martínez 2015) or even 1:3 for Singapore (Spieser et al. 2014). For the city of Berlin, the authors have conducted a study, which indicates that a fleet of 100000 vehicles is sufficient to serve the demand for private car trips during a weekday (Bischoff, Maciejewski 2016b). With the introduction of AT services, total vehicle mileage generally increases. For the Berlin case, the majority of extra traffic is generated in the outskirts of the city, leading to the overall conclusion that restricting AT services to densely populated city centre areas is more efficient (Bischoff, Maciejewski 2016a).

Research on AT services derives from previous studies on traditional taxi services, where with the advent of big data, GIS systems, and ICT technologies, the focus has moved from zone-based macroscopic models to large-scale microscopic simulations. In recent years, there have been many studies on multi-agent models of taxi services (Salanova et al. 2011; Salanova Grau 2013; Seow et al. 2010), centralised on-line taxi dispatching algorithms (Maciejewski et al. 2016; Zhan et al. 2016; Kümmel et al. 2016), or postprocessing GPS traces to analyse spatiotemporal characteristics of taxi demand and supply (Bischoff et al. 2015; Veloso et al. 2011; Li et al. 2011) or estimate urban travel times (Zhan et al. 2013; Ehmke et al. 2010). While in the short term, small AT fleets will operate in a similar way as their conventional counterparts, in the long term, however, large-scale AT services will differ significantly from taxi services of today. Because they are meant to replace private car traffic, the demand for AT services will be much different, in terms of both scale and spatiotemporal distribution, to that of today's taxis. Nowadays, for instance, the majority of rides starts and terminates within city centres and at airports (Bischoff et al. 2015; Salanova Grau, Estrada Romeu 2015) and the taxi demand also tends be the highest during weekend nights. Both cannot be expected for AT trips that focus on fulfilling everyday travel demand. Moreover, AT fleets made up of thousands of AVs will be managed fully automatically and in a centralised way, therefore instead of taxis waiting at ranks or roaming through the streets, we will observe relocation of empty taxis in anticipation of future demand and moving ATs outside the city centre in the off-peak hours. Although beyond the scope of this research, AT services may offer the option of sharing rides in order to reduce traffic, travel cost and/or the AT fleet size (Agatz et al. 2011; Fagnant, Kockelman 2015). 
The interaction of ATs and CDVs has not yet been part of many research studies we are aware of. All of the aforementioned studies consider travel times and AT dynamics to be equal to those of CDV traffic and assume similar congestion patterns. That approach, however, may be too simplistic, especially in scenarios where the whole public transport system is replaced, inducing much higher traffic. A recent study by (Levin et al. 2017) takes improved AV flow dynamics into account, however, the simulated scenario is limited in terms of size (only Austin's city centre) and spatial resolution (no intra-zonal trips). The results suggest twice as fast travel times during a morning peak using personal AVs and massive fleet reductions when sharing rides. A related problem of routing AVs in a capacitated transport network has recently been addressed in (Zhang et al. 2016). The study proposes an approach to real-time congestion-aware routing and rebalancing based on a network flow model. The method has been validated on a road network model of Manhattan using, however, a simple volume-delay function for modelling travel times.

\section{Methodology}

\subsection{MATSim}

MATSim (Horni et al. 2016b; Kickhöfer et al. 2016) is used to realistically simulate a large AT fleet together with normal traffic in the 3.5-million city of Berlin, Germany, where both types of participants try to optimise their performance given a limited flow capacity of the road network. MATSim combines a microscopic, disaggregated behavioural model of travellers with a mesoscopic queue-based traffic flow model and is capable of running large-scale simulations with millions of agents, e.g. (Erath et al. 2012) for Singapore.

The simulation is based on agents, which are equipped with (typically) day-long plans consisting of activities, such as home or work, and legs, where the mode and the route is defined. At the end of a day, plans are scored and, up to a certain point, modified for the next day (Horni et al. 2016a).

MATSim's traffic model is based on a queue model, which takes the flow capacity (throughput) and the storage capacity (equal to the length multiplied by the number of lanes) of a link in the network into account and moves vehicles according to the First-In First-Out (FIFO) scheme along the link: a car entering a network link is added to the tail of the link's waiting queue, where it remains until the time required to drive along the link under free flow conditions has passed. It is then allowed to leave the link, if it is the first car in the waiting queue. The latter allows as many cars to leave a link per time step as the flow capacity of a link suggests. For example, a flow capacity of 1200 vehicles/hour would allow one car every $3 \mathrm{~s}$ to leave the link. Leaving a link is, however, only possible if the storage capacity of the next link allows entering it (Rieser et al. 2016). Each vehicle uses the amount of flow and storage capacity, which corre- sponds to its length measured in Passenger Car Units (PCUs).

The queue model allows the simulation of millions of agents at high computational speeds. However, for analysis of traffic flow it brings certain limitations compared to more sophisticated, but also far slower models microscopic simulators (Maciejewski 2010), such as SUMO (Krajzewicz et al. 2012) or PTV VISSIM (Fellendorf, Vortisch 2010). First-of-all, vehicles in traffic are only tracked when entering and leaving a link. Secondly, traffic flow at intersections is modelled indirectly by flow capacities of incoming links and storage capacities of outgoing links; during each time step, vehicles waiting in front of the intersection are moved over it only if both constraints are not violated. In consequence, the only values that can be modified are aforementioned capacities. For this paper, the ability to compute large-scale scenarios was, however, found to be more important than the detail of the traffic flow simulation.

\subsection{Simulation of mixed AV/CDV traffic}

AVs are expected to increase road capacity; however, the actual improvement is hard to be precisely estimated. Recent studies suggest the improvement to be in the range of 1.5 and 2.0 for only-AV traffic, and in the case of mixed AV/CDV traffic, the relative improvement is expected to scale almost proportionally to the share of AVs (Wagner 2015; Levin et al. 2017; Friedrich 2015; Levin, Boyles 2016). In other words, an AV consumes 1.5 to 2.0 times less of the nominal flow capacity, measured in PCUs per hour, compared to a CDV of the same size, though they both occupy the same amount of space. To model that phenomenon, we have extended the vehicle model in MATSim with the flow capacity consumption coefficient so that flow and storage capacity consumption can be specified independently at the vehicle level.

As a result, more flexible handling of a link's flow capacity is possible: If, in the aforementioned example, $1200 \mathrm{veh} / \mathrm{h}$ are allowed to leave a link and all of them are CDVs, everything remains the same. If AVs only require half the amount of flow capacity of a CDV, 800 AVs travelling on the link during an hour would leave enough throughput for 800 additional CDVs. Thus, the actual flow capacity would be 1600 . If only AVs travel the link during an hour, the capacity would increase to 2400 vehicles. Figure 1 provides an illustration of the modified queue model and the increased throughput for mixed AV/CDV flow.

The capacity increase of a link can be written more formally. Let a mixed AV/CDV flow have a share $s \in[0 ; 1]$ of AVs, each of them consuming only a fraction $c \in(0 ; 1]$ of the amount of flow capacity consumed by a single CDV. Such a flow requires only a fraction $1-s+s c$ of the amount of flow capacity required by an equally intense CDV-only flow. As a result, the flow capacity increase ratio equals $1 /(1-s+s c)$. The impact of both parameters on the effective flow capacity increase is illustrated in Figure 2. 


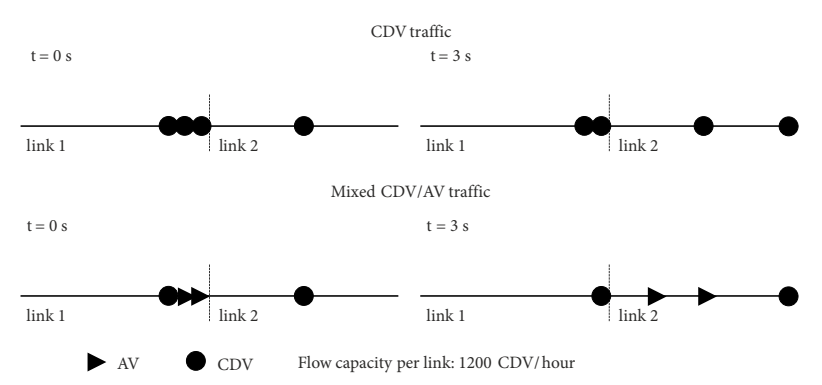

Figure 1. CDV and AV traffic dynamics in the adjusted queue model for two links at different times (the upper part shows only CDVs traversing the link, whereas the lower part includes both CDVs and AVs resulting in an increased throughput; in this example, an AV consumes only half the flow capacity a CDV does)

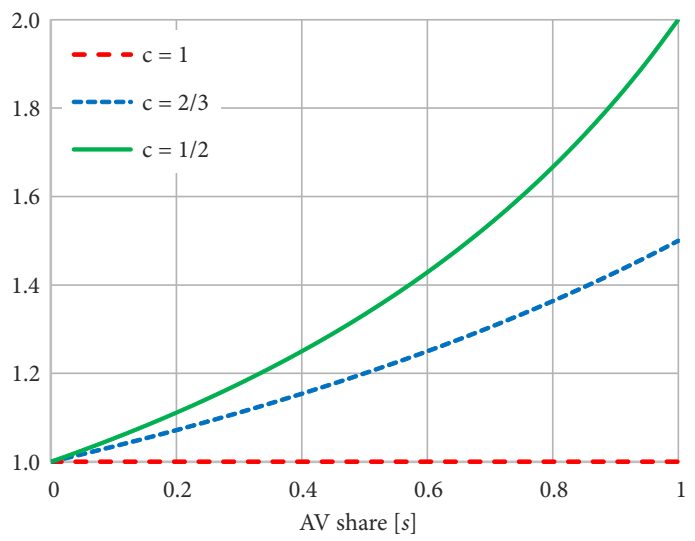

Figure 2. Flow capacity increase ratio of a link as a function of the AV share s for selected AV flow capacity consumption factors $c$

\subsection{Simulation of AT services}

Simulation of dynamic transport services in MATSim is handled by the Dynamic Vehicle Routing Problem (DVRP) module (Maciejewski 2016) that allows dynamic scheduling and routing of a fleet of vehicles in response to the current and anticipated demand. Quite recently, MATSim has been extended with a taxi module that offers many strategies for real-time dispatching of large taxi fleets (Maciejewski et al. 2016). The module has been already applied to simulate a 250000 strong AT fleet in Berlin (Bischoff, Maciejewski 2016b).

Typically, in MATSim, individuals (here people travelling by car or AT) pre-compute their daily plans and then they strictly follow the plan over the whole day (day-to-day re-planning). In order to simulate AT services, we modelled ATs as fully dynamic agents, who are managed centrally by the dispatching algorithm, and whose schedules can be changed at any moment in time (within-day re-planning).

In general, the dispatching algorithm reacts to incoming events (such as new request submissions, vehicle arrivals and departures) and dynamically re-optimises ATs' routes and schedules in order to ensure possibly most efficient execution of taxi orders. In this study, we use a simple yet very efficient and scalable rule-based strategy that handles undersupply and oversupply differ- ently in order to balance demand and supply. Typically, as long as there is at least one idle taxi, the nearest one is sent to each incoming request in order to minimise the passenger wait time. However, when all taxis are busy, all awaiting requests are stored, and once one of taxis becomes idle, it is sent to the nearest request, trying to maximize the system's throughput, which in turn, leads to minimisation of the average wait time.

\subsection{Simulation scenario}

A multi-modal MATSim model for Berlin has been used in several case studies and real-world transport planning (Neumann 2014; Kaddoura 2015). Its synthetic population depicts a typical workday in Berlin and the surrounding state of Brandenburg. Besides the full (100\%) scenario, which consists of roughly 6 million agents, other fractional (e.g. 10, 25\%) scenarios are often used. In this study, we use the $10 \%$ scenario, which is the most widely used.

Because the study explores the influence of Berlinwide AT services on traffic flow, the scenario was adapted so that only private car trips that start and/or end within the city area are taken into account, which makes up 508670 trips, out of which 278507 take place entirely within the city boundaries (Bischoff, Maciejewski 2016b). Computational experiments were conducted for six different replacement rates $(0,20, \ldots, 100 \%)$, where the rate defines the fraction of intra-city private car trips being converted into AT trips (cf. to Table 1). According to (Bischoff, Maciejewski 2016a), a fleet of 11000 AVs, each available 24 hours a day, is sufficient to serve all the private car trips while experiencing only a short period of undersupply during the afternoon peak. For smaller replacement rates, the fleet size is scaled down proportionally, for example, $2200 \mathrm{AVs}$ are used when only $20 \%$ of intra-city private car trips are converted into AT ones. The initial spatial distribution of ATs follows Berlin's population distribution.

Each computational experiment consists of 51 iterations, each being a simulation of a weekday day. In iteration 0 , all private car trips are routed (shortest path search) according to the link travel times obtained with the original (10\%) scenario. In iterations $1-40$, random $10 \%$ of private car trips are re-routed based on the link travel times from the previous iteration, which is aimed at reaching a dynamic equilibrium. In the last 10 iteration, private car routes are fixed. This is a standard approach in MATSim. However, during all 51 iterations,

Table 1. Replacement rate of car trips by AT mode and corresponding fleet sizes

\begin{tabular}{|c|c|c|}
\hline Replacement rate [\%] & AT trips & AT fleet \\
\hline 0 & 0 & 0 \\
\hline 20 & 55701 & 2200 \\
\hline 40 & 111403 & 4400 \\
\hline 60 & 167104 & 6,600 \\
\hline 80 & 222806 & 8800 \\
\hline 100 & 278507 & 11000 \\
\hline
\end{tabular}


ATs are dispatched always along the shortest path, which can lead to day-to-day oscillations when the link travel times are taken only from the previous day. In order to prevent that and reach an equilibrium, travel times used for AT dispatching are determined by applying an exponential moving average (over travel times observed over a series of iterations) with a relatively low degree of weighting decrease $(\alpha=0.05)$. This approach could be extended with short-term on-line travel time prediction techniques proposed by (Hörl 2016).

Because traffic flow is simulated for the $10 \%$ scenario, the link flow and storage capacities are multiplied by 0.15 and 0.3 , respectively. The reasons behind not using 0.1 scaling factors are twofold: the travel demand is around $11 \%$ (not 10\%) of that in the full scenario, and secondly, granularity of traffic flow prevents simple linear capacity downscaling, in particular for smaller links. Although AVs are expected to improve traffic flow, the scale of improvement still remains an open question. Therefore, computations were carried out for three levels of capacity increase given an AV-only flow $(s=1)$, namely 1.0 (no improvement; $c=1), 1.5(c=2 / 3)$, and 2.0 (twofold increase; $c=1 / 2$ ), all three presented in Figure 2. While referring to specific simulation cases (scenario variations), we often use abbreviations that combine the applied replacement rate and the capacity increase level, for example ' $0 \%$ ' (no ATs) or ' $100 \%, 1.0$ ' (all intra-city trips handled by ATs and no improvement in $\mathrm{AV}$ flow dynamics).

In order to speed up computations, the nearest open request or idle vehicles is found with multi-node Dijkstra search out of the 20 nearest (in a straight line) open request or idle vehicles, respectively. Each pickup takes 2 minutes and each drop-off 1 minute.

Computations were run in parallel (one simulation experiment per physical core) on a computer with a 6-core Intel Core i7-3930K Processor (12 logical cores) and 64GB RAM. Each iteration consisted mainly of the following two phases: re-planning (including re-routing of $10 \%$ private car trips; up to iteration 40 ), which took on average between 250 and $400 \mathrm{~s}$, and simulation (of both traffic flow and AT dispatching), which required from 400 (the $0 \%$ case, when only traffic is simulated) to $2000 \mathrm{~s}(100 \%, 1.0)$.

a)

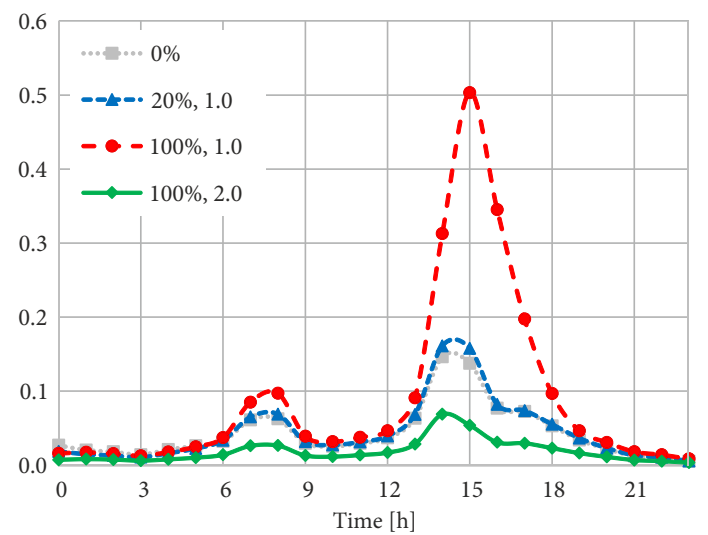

\section{Results}

\subsection{Traffic congestion}

To assess the impact of the AT service on traffic congestion, we calculated the average travel time for all intra-city trips excluding empty drives made by ATs (i.e. occupied AT drives and private car trips) beginning in a given time period (e.g. hour or day). This indicator allows for comprehensive assessment of the total impact on intra-city travellers, regardless of the mode chosen. In order to compare the level of congestion at any hour, we also calculated delay ratios, defined as the average travel delay (the difference between the actual and free-flow travel times) to the average free-flow (uncongested) travel time. Table 2 shows the indicator values obtained for the afternoon peak hour (3:00-4:00 pm) and the whole day. Figure 3a compares hourly delay ratios over the day for selected scenario variations, whereas Figure $3 \mathrm{~b}$ illustrates the afternoon peak hour ratios for all variations.

The differences in delays between analysed cases are especially noticeable during peak hours and at high replacement rates. While the results for the $20 \%$ replacement rate are close to the $0 \%$ case, regardless of the capacity increase level, the full replacement of private cars with ATs leads to significant changes in traffic. In the latter case, when there is no increase in road capacity, additional empty taxi trips induce significant congestion. However, the capacity increase level of 1.5 more than compensates for unoccupied taxi travel. This level of flow improvement is enough to reduce traffic congestion. Further increase of the factor does not bring substantial changes.

It should be noted that the calculated delay ratios are underestimated, and seem moderate even in the most extreme case $(100 \%, 1.0)$. Firstly, in real life, vehicles typically move faster in free flow than the allowed maximal speed, which is not taken into account in this study. Secondly, Berlin is not as heavily congested as other cities of similar size.

Another look at traffic situation is provided by daily profiles of the number of vehicles driving in the network. Figure 4a shows profiles generated for all vehicles, whereas Figure $4 \mathrm{~b}$ refers only to vehicles on intra-city trips (including both empty and occupied AT drives).

b)

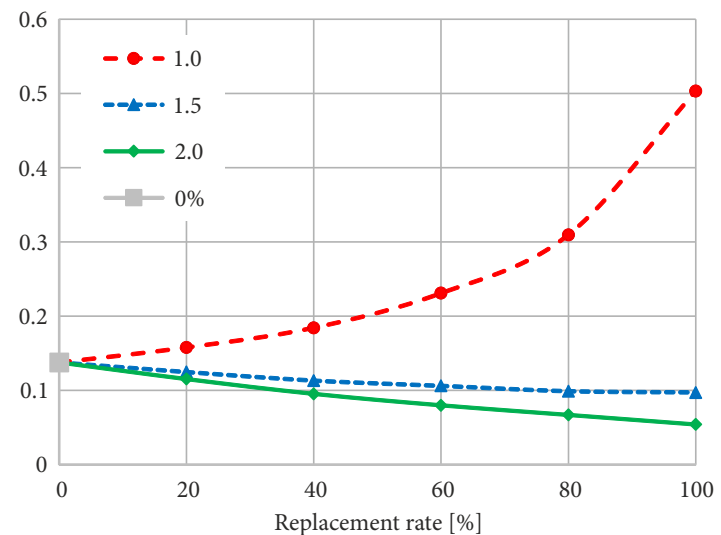

Figure 3. Travel delay ratios: a - hourly averages for selected simulation cases; b - peak-hour averages extrapolated to the $0 \%$ replacement case (grey point) 
Table 2. Mean travel time and delay ratio for all intra-city trips (excluding empty taxi drives)

\begin{tabular}{|c|c|c|c|c|c|}
\hline \multirow{2}{*}{ Replacement rate [\%] } & \multirow{2}{*}{ Capacity increase level } & \multicolumn{2}{|c|}{$3: 00-4: 00 \mathrm{pm}$} & \multicolumn{2}{|c|}{ All day } \\
\hline & & Travel time [s] & Delay ratio [\%] & Travel time [s] & Delay ratio [\%] \\
\hline \multicolumn{2}{|c|}{ Free flow } & 703 & - & 716 & - \\
\hline 0 & - & 799 & 13.8 & 763 & 6.5 \\
\hline \multirow{3}{*}{20} & 1.0 & 814 & 15.8 & 767 & 7.1 \\
\hline & 1.5 & 790 & 12.5 & 759 & 6.0 \\
\hline & 2.0 & 784 & 11.5 & 756 & 5.6 \\
\hline \multirow{3}{*}{40} & 1.0 & 832 & 18.4 & 772 & 7.8 \\
\hline & 1.5 & 782 & 11.3 & 756 & 5.5 \\
\hline & 2.0 & 770 & 9.5 & 750 & 4.8 \\
\hline \multirow{3}{*}{60} & 1.0 & 865 & 23.1 & 780 & 8.9 \\
\hline & 1.5 & 777 & 10.6 & 753 & 5.1 \\
\hline & 2.0 & 759 & 8.0 & 745 & 4.1 \\
\hline \multirow{3}{*}{80} & 1.0 & 920 & 31.0 & 797 & 11.2 \\
\hline & 1.5 & 772 & 9.9 & 750 & 4.8 \\
\hline & 2.0 & 750 & 6.7 & 741 & 3.4 \\
\hline \multirow{3}{*}{100} & 1.0 & 1056 & 50.3 & 828 & 15.7 \\
\hline & 1.5 & 771 & 9.7 & 749 & 4.5 \\
\hline & 2.0 & 741 & 5.4 & 736 & 2.8 \\
\hline
\end{tabular}

a)

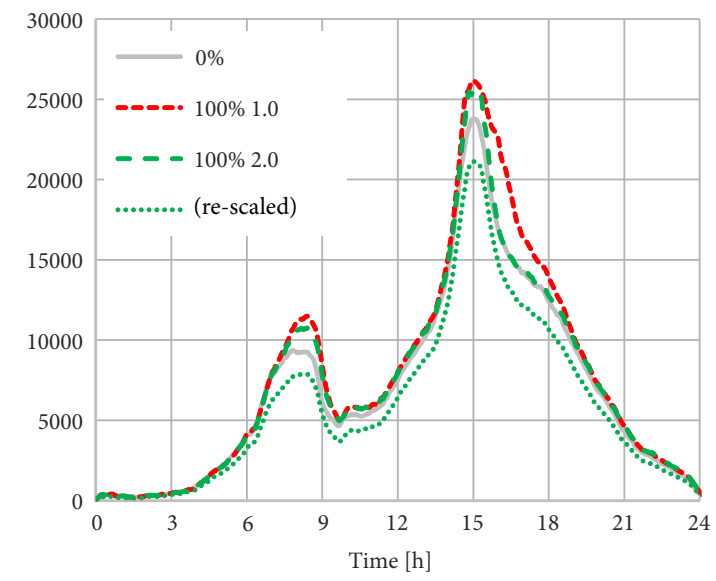

b)

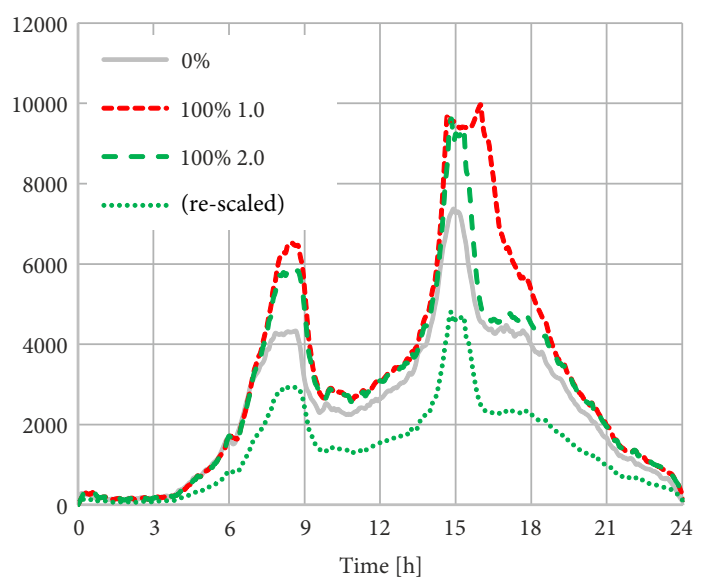

Figure 4. Number of vehicles driving in the network: a - all trips; b - intra-city trips (the dotted green curve is a re-scaled $100 \%$, 2.0 series, where each $\mathrm{AV}$ is counted as $0.5 \mathrm{CDV}$ s to adjust for their reduced flow capacity consumption)

Globally, there is not so much difference between the illustrated scenario variations, as many trips incoming to and outgoing from Berlin are spread over a larger area, and thus, remain unaffected by the AT fleet. However, taking only vehicles on intra-city trips into account gives more insight into the places where traffic is most intensive, such as the city centre. Serving all intra-city trips by AT increases the number of vehicles in the network by around two thousand because of empty drives between consecutive taxi requests. However, this effect is limited by the fleet size: around 3:00 pm up to ten thousands of ATs are driving in the network while the remaining one thousand is picking up and dropping off passengers.

Despite the additional mileage triggered by dispatch of empty taxis, the increase in flow capacity, particularly of those links which are often traversed by AVs, significantly lowers congestion. The dotted green curve in both charts represents the normalised number of vehicles in the network, where each AT is counted as 0.5 CDVs, which approximates the network-wide demand for flow capacity in the ' $100 \%, 2.0$ ' scenario variation. At the opposite extreme, serving all intra-city trips with ATs given no increase in road capacity, leads to severe traffic congestion resulting in the afternoon peak being extended by additional two hours.

Because AT operation is concentrated within the city centre, this is also the area which is impacted most by the introduction of ATs. A deeper insight into a spatial effects is given in Figure 5 that shows differences in the peak-hour link speed ratios (defined as the ratio of peak-hour to free-flow speed) between the no AT (0\%) case and the full replacement with no AV flow improve- 


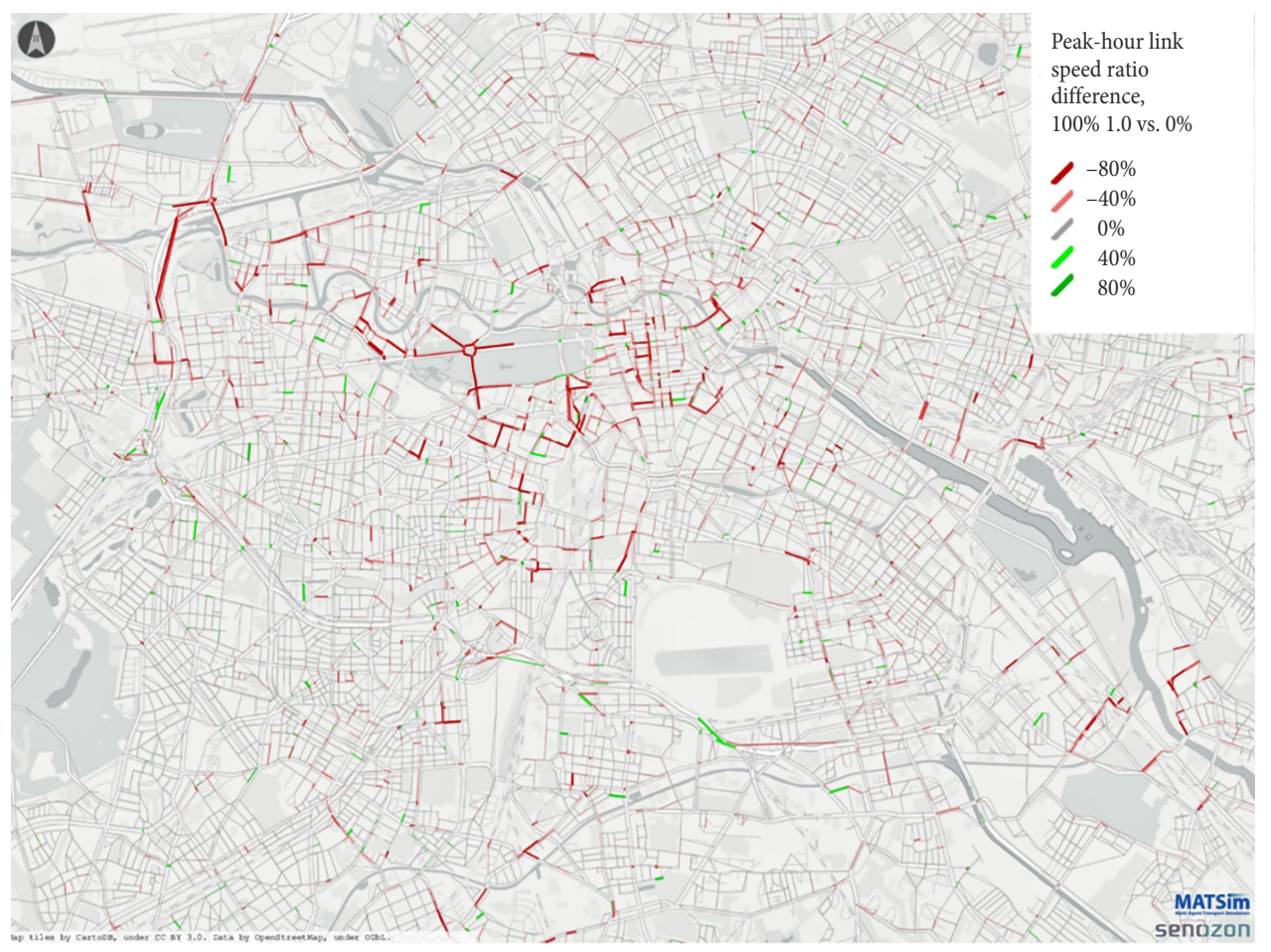

Figure 5. Differences in the link speed ratios on links during the pm peak hour between the full replacement with no AV flow improvement $(100 \%, 1.0)$ case and the no AT $(0 \%)$ case (additional empty rides result in an overall decrease of network speed, marked in red)

ment $(100 \%, 1.0)$ case. A relative drop in speed of up to $80 \%$ can be noticed on links in the city centre, marking a significant increase in congestion. This is mainly an issue on arterial roads and parts of the circular motorway. Because of stochasticity, some links are also less congested (marked in green).

\subsection{AT service performance}

Performance of the AT service can be assessed from different points of view. Passengers want a high quality service (e.g. low waiting times), but at the same time, the dispatcher wants to minimise operation costs (e.g. a low empty to total drive time ratio for short-term planning, or a small fleet size for long-term planning). Table 3 and Figures 6-8 show selected results obtained for the following three measures: the average passenger wait time for trips starting within a given period, the average AT utilisation rate in a given period, and the average vehicle-wise (as opposed to trip-wise) empty to total drive time ratio over a given period. Since the fleet size was fixed, the utilisation rate is used not as a service performance indicator, but only to assess the impact of traffic on AT operation.

Table 3. AT fleet performance

\begin{tabular}{|c|c|c|c|c|c|c|c|}
\hline \multirow{2}{*}{$\begin{array}{l}\text { Replacement } \\
\text { rate }[\%]\end{array}$} & \multirow{2}{*}{$\begin{array}{c}\text { Capacity increase } \\
\text { level }\end{array}$} & \multicolumn{2}{|c|}{ Passenger wait time $[\mathrm{s}]$} & \multicolumn{2}{|c|}{ AT utilisation rate [\%] } & \multicolumn{2}{|c|}{ AT empty drive ratio [\%] } \\
\hline & & 3:00-4:00 pm & all day & $3: 00-4: 00 \mathrm{pm}$ & all day & $3: 00-4: 00 \mathrm{pm}$ & all day \\
\hline \multirow{3}{*}{20} & 1.0 & 334 & 190 & 95.5 & 31.6 & 22.6 & 18.8 \\
\hline & 1.5 & 281 & 183 & 92.6 & 31.2 & 20.4 & 18.6 \\
\hline & 2.0 & 286 & 182 & 92.5 & 31.2 & 21.8 & 18.7 \\
\hline \multirow{3}{*}{40} & 1.0 & 285 & 186 & 93.9 & 31.5 & 16.7 & 17.7 \\
\hline & 1.5 & 230 & 174 & 89.4 & 30.9 & 16.9 & 17.8 \\
\hline & 2.0 & 218 & 172 & 88.0 & 30.8 & 16.8 & 17.8 \\
\hline \multirow{3}{*}{60} & 1.0 & 314 & 185 & 96.7 & 31.5 & 16.9 & 17.0 \\
\hline & 1.5 & 214 & 166 & 89.3 & 30.6 & 16.6 & 17.1 \\
\hline & 2.0 & 191 & 161 & 86.7 & 30.4 & 16.1 & 17.1 \\
\hline \multirow{3}{*}{80} & 1.0 & 374 & 195 & 98.5 & 32.0 & 17.6 & 16.9 \\
\hline & 1.5 & 196 & 163 & 88.4 & 30.5 & 15.9 & 16.9 \\
\hline & 2.0 & 167 & 156 & 84.8 & 30.1 & 15.3 & 16.9 \\
\hline \multirow{3}{*}{100} & 1.0 & 483 & 212 & 99.9 & 33.1 & 15.9 & 16.8 \\
\hline & 1.5 & 194 & 163 & 89.1 & 30.4 & 16.9 & 16.9 \\
\hline & 2.0 & 153 & 153 & 83.9 & 29.9 & 15.0 & 16.7 \\
\hline
\end{tabular}


a)

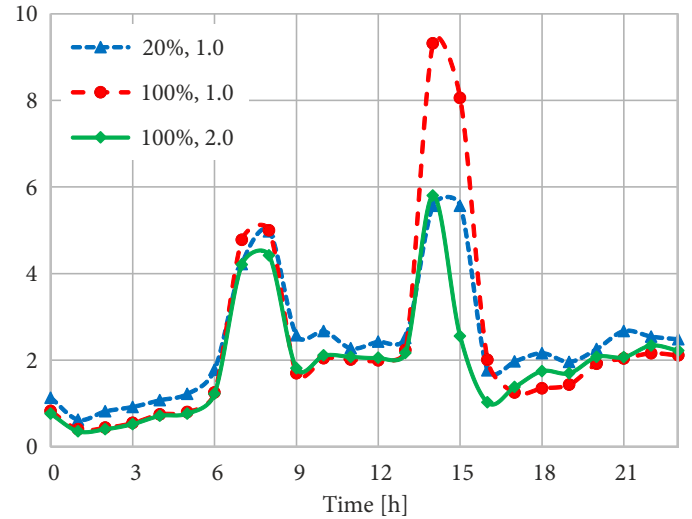

b)

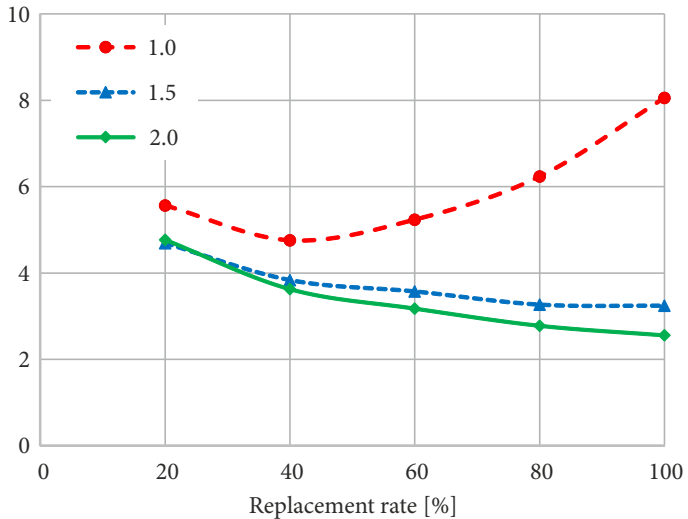

Figure 6. Passenger wait times: a - hourly averages for selected simulation cases; $b$ - peak-hour averages

a)

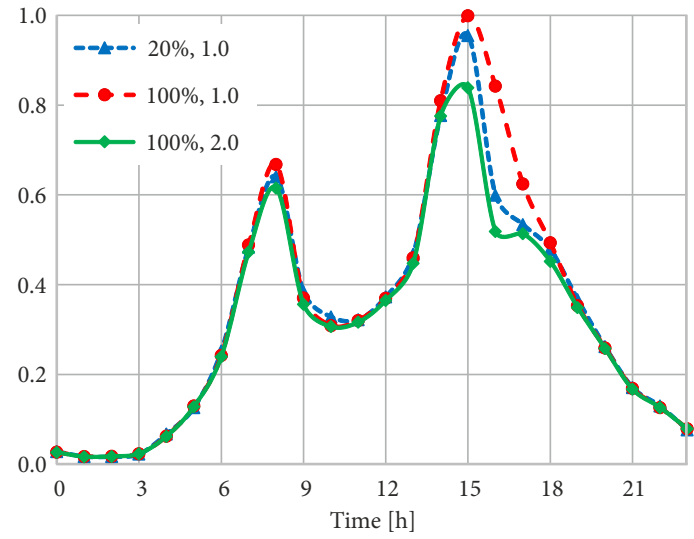

b)

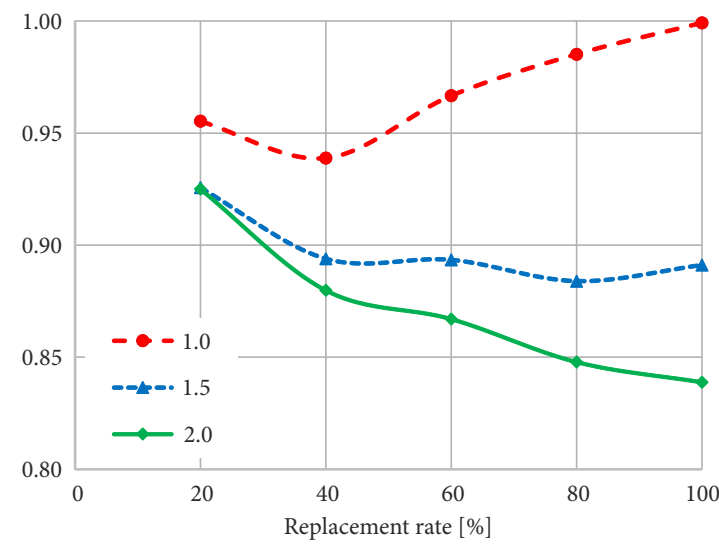

Figure 7. AT utilisation rates: $\mathrm{a}$ - hourly averages for selected simulation cases; $\mathrm{b}$ - peak-hour averages

a)

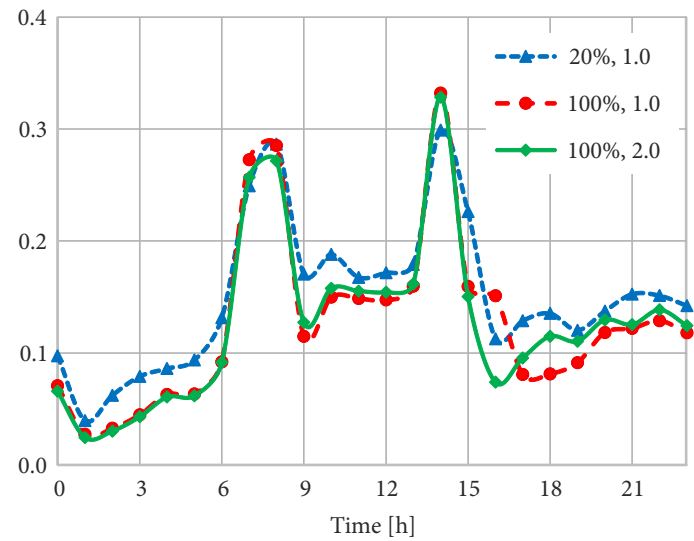

b)

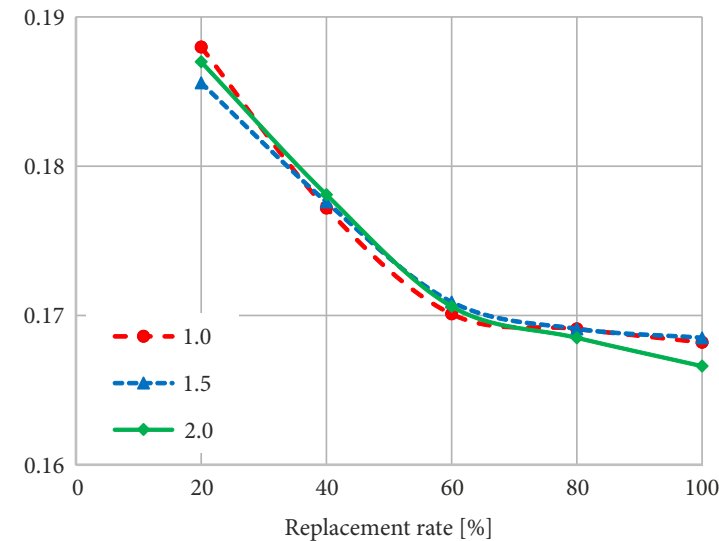

Figure 8. AT empty drive ratios: a - hourly averages for selected simulation cases; b - daily averages

Analysis of taxi service performance during the afternoon peak gives a clear view on the AT service under congestion. If the AV flow is assumed to be identical to the CDV flow (the capacity increase level of 1.0), the best peak-hour performance (the lowest passenger wait time and AT utilisation rate) is obtained for the $40 \%$ replacement rate, which is a trade-off between having a larger (effect of scale resulting in shortening empty drives) or smaller fleet (less congestion due to fewer empty drives). On the opposite pole, once AV flow dynamics is improved (the capacity increase levels of 1.5 and 2.0), the overall traffic gets more fluid, which in turns significantly increases the AT service performance, becoming highest at the $100 \%$ replacement rate. All-inall, higher capacity more than compensates extra traffic generated by empty drives.

The only performance measure that does not depend so much on traffic condition and is mostly impacted by the fleet size (effect of scale) is the empty drive ratio. Intuitively, one could expect higher empty trip ratios in congested scenarios because fewer vehicles remain available for dispatch, causing them to travel longer distances to reach customers. However, when the systems runs into undersupply, the dispatching strategy actually starts minimising empty drive time, as opposed to minimising passenger wait time during oversupply. 


\section{Conclusions}

The obtained results suggest that operating large AT fleets in cities may have a positive effect on traffic. Even the moderate AV flow capacity increase of 1.5 , which is a quite low estimate given the state-of-the-art predictions, reduces congestion at all replacement ratios, despite increased traffic volumes caused by empty AT drives. A further capacity increase from 1.5 to 2.0 leads to practically uncongested traffic at the $100 \%$ replacement rate. AT operation is especially beneficial in the congested city centre, where empty mileage is relatively low due to high density of the AT demand and supply. While moving away from the centre, ATs generate more empty taxi traffic and the overall impact is less substantial, yet still positive. However, if AVs do not effect in higher road capacity, AT services cannot be introduced on a large scale, as that would lead to even heavier congestion in urban areas. Because the effects of capacity increase are variable in space and time, it is hard to approximate a borderline value of the capacity increase level that would balance out the negative impact of empty trips. This question requires further computational experiments. Also a possible improvement of CDVs, e.g. by (retro-) fitting some of the features of AVs such as platooning, should be looked at closer.

The transition phase, with only few ATs operative and a majority of CDVs remaining in the city, will not trigger significantly more congestion even if road capacity remains at the same level. However, a smaller AT fleet will result in a poorer dispatch performance due to higher empty mileage. For that period, a smaller operation area, such as the city centre, may be worth considering.

Empty AT traffic results in the overall traffic volume increase. Ride sharing could be a way to reduce this effect, especially during high peak periods in the city centre. Another potential benefit of ride sharing is a smaller AT fleet, which is sized for the peak demand and remains rather idle for most of the day. Yet, it is unclear in how far ride sharing is accepted by current car users and it is certainly not a solution when AT demand is low, especially in sparsely populated areas and at night. In this case, combining public transport with ATs serving last-mile trips is likely an approach to follow. These two issues of sharing rides and integration with public transport, should be addressed in future studies.

\section{References}

Agatz, N. A. H.; Erera, A. L.; Savelsbergh, M. W. P.; Wang, X. 2011. Dynamic ride-sharing: a simulation study in metro Atlanta, Transportation Research Part B: Methodological 45(9): 1450-1464. https://doi.org/10.1016/j.trb.2011.05.017

Bischoff, J.; Maciejewski, M. 2016a. Autonomous taxicabs in Berlin - a spatiotemporal analysis of service performance, Transportation Research Procedia 19: 176-186. https://doi.org/10.1016/j.trpro.2016.12.078

Bischoff, J.; Maciejewski, M. 2016b. Simulation of city-wide replacement of private cars with autonomous taxis in Berlin, Procedia Computer Science 83: 237-244. https://doi.org/10.1016/j.procs.2016.04.121
Bischoff, J.; Maciejewski, M.; Sohr, A. 2015. Analysis of Berlin's taxi services by exploring GPS traces, in 2015 International Conference on Models and Technologies for Intelligent Transportation Systems (MT-ITS), 3-5 June 2015, Budapest, Hungary, 209-215.

https://doi.org/10.1109/MTITS.2015.7223258

Burns, L. D.; Scarborough, B. A. 2013. Transforming Personal Mobility. The Earth Institute, Columbia University, New York, NY, US. 43 p. Available from Internet: http://sustainablemobility.ei.columbia.edu/files/2012/12/TransformingPersonal-Mobility-Jan-27-20132.pdf

Ehmke, J. F.; Meisel, S.; Mattfeld, D. C. 2010. Floating car data based analysis of urban travel times for the provision of traffic quality, International Series in Operations Research \& Management Science 144: 129-149.

https://doi.org/10.1007/978-1-4419-6070-2_9

Erath, A.; Fourie, P.; Van Eggermond, M.; Ordóñez, S.; Chakirov, A.; Axhausen, K. 2012. Large-scale agent-based transport demand model for Singapore. Working Paper. Institute for Transport Planning and Systems, Swiss Federal Institute of Technology Zurich. 40 p. Available from Internet: https://www.ethz.ch/content/dam/ethz/special-interest/baug/ ivt/ivt-dam/vpl/reports/701-800/ab790.pdf

Fagnant, D. J; Kockelman, K. M. 2015. Dynamic Ride-Sharing and Optimal Fleet Sizing for a System of Shared Autonomous Vehicles, in Transportation Research Board 94th Annual Meeting, 11-15 January 2015, Washington, DC, US, 1-17.

Fajardo, D.; Au, T.-C.; Waller, S.; Stone, P.; Yang, D. 2011. Automated intersection control: performance of future innovation versus current traffic signal control, Transportation Research Record: Journal of the Transportation Research Board 2259: 223-232. https://doi.org/10.3141/2259-21

Fellendorf, M.; Vortisch, P. 2010. Microscopic traffic flow simulator VISSIM, International Series in Operations Research \& Management Science 145: 63-93. https://doi.org/10.1007/978-1-4419-6142-6_2

Friedrich, B. 2015. Verkehrliche Wirkung autonomer Fahrzeuge, in M. Maurer, J. C. Gerdes, B. Lenz, H. Winner (Eds.). Autonomes Fahren, 331-350.

https://doi.org/10.1007/978-3-662-45854-9_16 (in German).

Hörl, S. 2016. Implementation of an autonomous taxi service in a multi-modal traffic simulation using MATSim: Master thesis in Complex Adaptive Systems. Chalmers University of Technology, Gothenburg, Sweden. 90 p. Available from Internet: http://publications.lib.chalmers.se/records/fulltext/237733/237733.pdf

Horni, A.; Nagel, K.; Axhausen, K. W. 2016a. Introducing MATSim, in A. Horni, K. Nagel, K. W. Axhausen (Eds.). The Multi-Agent Transport Simulation MATSim, 3-8.

Horni, A.; Nagel, K.; Axhausen, K. W. 2016b. The Multi-Agent Transport Simulation MATSim. Ubiquity Press Ltd. 620 p. https://doi.org/10.5334/baw

Kaddoura, I. 2015. Marginal congestion cost pricing in a multiagent simulation: investigation of the greater Berlin area, Journal of Transport Economics and Policy 49(4): 560-578.

Kickhöfer, B.; Hosse, D.; Turner, K.; Tirachini, A. 2016. Creating an Open MATSim Scenario from Open Data: the Case of Santiago de Chile. VSP Working Paper 16-02. Technical University of Berlin, Germany. 22 p. Available from Internet: http://svn.vsp.tu-berlin.de/repos/public-svn/publications/vspwp/2016/16-02/KickhoeferEtAl2016MatsimSantiago.pdf 
Krajzewicz, D.; Erdmann, J.; Behrisch, M., Bieker, L. 2012. Recent development and applications of SUMO - Simulation of Urban MObility, International Journal on Advances in Systems and Measurements 5(3\&4): 128-138.

Levin, M. W.; Boyles, S. D. 2016. A multiclass cell transmission model for shared human and autonomous vehicle roads, Transportation Research Part C: Emerging Technologies 62: 103-116. https://doi.org/10.1016/j.trc.2015.10.005

Levin, M. W.; Kockelman, K. M.; Boyles, S. D.; Li, T. 2017. A general framework for modeling shared autonomous vehicles with dynamic network-loading and dynamic ridesharing application, Computers, Environment and Urban Systems 64: 373-383. https://doi.org/10.1016/j.compenvurbsys.2017.04.006

Li, B.; Zhang, D.; Sun, L.; Chen, C.; Li, S.; Qi, G.; Yang, Q. 2011. Hunting or waiting? Discovering passenger-finding strategies from a large-scale real-world taxi dataset, in 2011 IEEE International Conference on Pervasive Computing and Communications Workshops (PERCOM Workshops), 21-25 March 2011, Seattle, WA, US, 63-68. https://doi.org/10.1109/PERCOMW.2011.5766967

Litman, T. 2015. Autonomous vehicle implementation predictions: implications for transport planning, in Transportation Research Board 94th Annual Meeting, 11-15 January 2015, Washington, DC, US, 1-15.

Kümmel, M.; Busch, F.; Wang, D. Z. W. 2016. Taxi dispatching and stable marriage, Procedia Computer Science 83: 163170. https://doi.org/10.1016/j.procs.2016.04.112

Maciejewski, M. 2016. Dynamic transport services, in A. Horni, K. Nagel, K. W. Axhausen (Eds.). The Multi-Agent Transport Simulation MATSim, 145-152.

Maciejewski, M. 2010. A comparison of microscopic traffic flow simulation systems for an urban area, Transport Problems - Problemy Transportu 5(4): 27-38.

Maciejewski, M.; Bischoff, J.; Nagel, K. 2016. An assignmentbased approach to efficient real-time city-scale taxi dispatching, IEEE Intelligent Systems 31(1): 68-77. https://doi.org/10.1109/MIS.2016.2

Martínez, L. 2015. Urban Mobility System Upgrade: How Shared Self-Driving Cars Could Change City Traffic. Corporate Partnership Board Report. International Transport Forum. 36 p. Available from Internet: https://www.itf-oecd. $\mathrm{org} /$ sites/default/files/docs/15cpb_self-drivingcars.pdf

Neumann, A. 2014. A Paratransit-Inspired Evolutionary Process for Public Transit Network Design: PhD Dissertation. Technical University of Berlin, Germany. 267 p.

Rieser, M.; Nagel, K.; Horni, A. 2016. MATSim data containers, in A. Horni, K. Nagel, K. W. Axhausen (Eds.). The Multi-Agent Transport Simulation MATSim, 55-60.

Salanova, J. M.; Estrada, M. A.; Aifadopoulou, G.; Mitsakis, E. 2011. A review of the modeling of taxi services, Procedia Social and Behavioral Sciences 20: 150-161. https://doi.org/10.1016/j.sbspro.2011.08.020

Salanova Grau, J. M.; Estrada Romeu, M. A. 2015. Agent based modelling for simulating taxi services, Procedia Computer Science 52: 902-907. https://doi.org/10.1016/j.procs.2015.05.162

Salanova Grau, J. M.; Estrada Romeu, M. A., Mitsakis, E.; Stamos, I. 2013. Agent based modeling for simulation of taxi services, Journal of Traffic and Logistics Engineering 1(2): 159-163. https://doi.org/10.12720/jtle.1.2.159-163
Seow, K. T.; Dang, N. H.; Lee, D.-H. 2010. A collaborative multiagent taxi-dispatch system, IEEE Transactions on Automation Science and Engineering 7(3): 607-616. https://doi.org/10.1109/TASE.2009.2028577

Spieser, K.; Treleaven, K.; Zhang, R.; Frazzoli, E.; Morton, D.; Pavone, M. 2014. Toward a systematic approach to the design and evaluation of automated mobility-on-demand systems: a case study in Singapore, in G. Meyer, S. Beiker (Eds.). Road Vehicle Automation, 229-245. https://doi.org/10.1007/978-3-319-05990-7_20

Van Arem, B.; Van Driel, C. J. G.; Visser, R. 2006. The Impact of Cooperative Adaptive Cruise Control on Traffic-Flow Characteristics, IEEE Transactions on Intelligent Transportation Systems 7(4): 429-436. https://doi.org/10.1109/TITS.2006.884615

Veloso, M.; Phithakkitnukoon, S; Bento, C. 2011. Sensing urban mobility with taxi flow, in LBSN'11: Proceedings of the 3rd ACM SIGSPATIAL International Workshop on Location-Based Social Networks, 1-4 November 2011, Chicago, US, 41-44. https://doi.org/10.1145/2063212.2063215

Wadud, Z.; MacKenzie, D.; Leiby, P. 2016. Help or hindrance? The travel, energy and carbon impacts of highly automated vehicles, Transportation Research Part A: Policy and Practice 86: 1-18. https://doi.org/10.1016/j.tra.2015.12.001

Wagner, P. 2015. Steuerung und Management in einem Verkehrssystem mit autonomen Fahrzeugen, in M. Maurer, J. C. Gerdes, B. Lenz, H. Winner (Eds.). Autonomes Fahren, 313-330. https://doi.org/10.1007/978-3-662-45854-9_15 (in German).

Zhan, X.; Hasan, S.; Ukkusuri, S. V.; Kamga, C. 2013. Urban link travel time estimation using large-scale taxi data with partial information, Transportation Research Part C: Emerging Technologies 33: 37-49. https://doi.org/10.1016/j.trc.2013.04.001

Zhan, X.; Qian, X.; Ukkusuri, S. V. 2016. A graph-based approach to measuring the efficiency of an urban taxi service system, IEEE Transactions on Intelligent Transportation Systems 17(9): 2479-2489.

https://doi.org/10.1109/TITS.2016.2521862

Zhang, R.; Rossi, F.; Pavone, M. 2016. Routing autonomous vehicles in congested transportation networks: structural properties and coordination algorithms, Robotics: Science and Systems 12: 1-9.

https://doi.org/10.15607/RSS.2016.XII.032 